\title{
Magnetic scattering effects on quantum transport in a normal-metal-superconductor junction
}

\author{
Jian-Xin Zhu and Z. D. Wang \\ Department of Physics, University of Hong Kong, Pokfulam Road, Hong Kong \\ (Received 20 September 1996; revised manuscript received 1 November 1996)
}

\begin{abstract}
Within a scattering formalism, the magnetic scattering effects on the transport properties of a normal metal attached to a $d$-wave as well as an $s$-wave superconductor have been studied by inserting (i) a Kondo-like magnetic barrier and (ii) a ferromagnetic scattering layer into the normal conducting region. It is shown that, in contrary to our intuition, the Kondo-like magnetic scattering effect is quite similar to the effect of nonmagnetic scattering. Remarkably, the ferromagnetic exchange interaction could lead to the strong resemblance of the conductance behavior between a normal-metal-s-wave superconductor junction containing a ferromagnet layer and a normal-metal- $d$-wave superconductor with a nonmagnetic scattering layer, and vice versa. This result may complicate significantly the decisive determination of the pairing symmetry in high- $T_{c}$ superconductors by the quasiparticle tunneling into the superconductor. In addition, the resonance peak splitting in the conductance is exhibited if the ferromagnet layer is located several superconducting coherence lengths away from the normal-metal-superconductor interface.
\end{abstract}

[S0163-1829(97)02913-5]

\section{INTRODUCTION}

With the recent advance in nanotechnology, interest in quantum transport through mesoscopic normal metals or semiconductors coupled with superconductors has been renewed due to possible applications in electronic devices. ${ }^{1}$ In these hybrid systems, electrons are not only intrinsically coherent in the superconducting region but also retain phase memory throughout the normal conducting region. At the interface between the normal conductor and the superconductor, a scattering process known as the Andreev reflection ${ }^{2}$ occurs: An electron incident from the normal conductor with an energy below the superconducting energy gap cannot drain off into the superconductor. It is instead reflected from the normal-metal-superconductor (NS) interface as a hole by transferring a charge $-2 e(e>0)$ to the superconductor. In addition, if a tunnel barrier is introduced into an otherwise ballistic normal-metal-superconductor junction, electrons are always reflected from the barrier as electrons, rather than as holes. There have been many theoretical and experimental efforts devoted to the current-voltage $(I-V)$ characteristic of normal-metal-isotropic $s$-wave superconductor junctions. ${ }^{3}$ The coherent scattering between the pair potential of the superconductor and the ordinary electrostatic potential of a tunnel barrier modifies the wave interference pattern and thus changes the $I-V$ characteristic. Recently, the pairing symmetry of high- $T_{c}$ superconductors has also received much attention, and many theoretical and experimental studies ${ }^{4}$ have suggested that the pairing state of cuprate high- $T_{c}$ supercon-

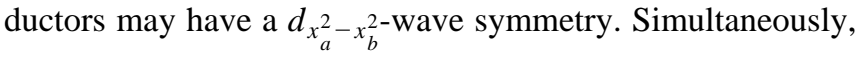
investigations of the $I-V$ characteristic have been extended to consider normal-metal- $d$-wave superconductor junctions. ${ }^{5-7}$ It is found that the conductance zero-bias anomaly (ZBA) observed in normal-metal-high- $T_{c}$ superconductor junctions ${ }^{8}$ could be attributed ${ }^{5,6}$ to the existence of midgap states $^{9}$ when the order parameter of the superconductor is of $d$-wave symmetry which gives rise to the sign change of the anisotropic energy gap across some nodal points of the Fermi surface. In this case, the tunnel barrier resides at the NS interface and the normal metal is ballistic. When the tunnel barrier resides in the ballistic normal metal several superconducting coherence lengths away from the interface, subgap resonances occur as those in conventional NS junctions except a midgap resonance at zero voltage. ${ }^{6}$ So far, most studies have dealt with the nonmagnetic scattering. Since the Andreev reflection near the Fermi level conserves energy and momentum but does not conserve spin, that is, the incoming electron and the Andreev-reflected hole occupy opposite spin bands, this effect is irrelevant for the spin-independent scattering. However, when the magnetic scattering is involved in the transport of electrons through a normal-metalsuperconductor junction, due to the lack of spin conservation associated with the Andreev reflection, novel behavior may appear in conductance. Motivated in part by this observation, in this paper, we study the magnetic scattering effects by considering (i) a sheet Kondo-like magnetic barrier and (ii) a magnetic insulator inserted into the normal conducting region, while taking the pairing symmetry of the attached superconductor to be either $s$ wave or $d$ wave. The conductance is calculated by generalizing the previous theory of Blonder, Tinkham, and Klapwijk ${ }^{10}$ (BTK) to include the spin effect. It is found that, the ferromagnetic scattering could lead to the strong resemblance of the conductance behavior between a normal-metal-s-wave superconductor junction containing a ferromagnet layer and a normal-metal- $d$-wave superconductor with a nonmagnetic scattering layer, and vice versa. This result shows that the explanation of the ZBA observed in high- $T_{c}$ superconductor junctions, which is based on the $d$-wave pairing, may not be unique.

The paper is organized as follows. Section II presents the Bogoliubov-de Gennes equations for unconventional superconductors and the reduced form we will use. In Sec. III, we calculate the conductance of $s$-wave and $d$-wave junctions both containing a Kondo-like magnetic barrier. The results for the conductance of NS junctions with a ferromagnetic 
scattering layer is given in Sec. IV. Finally, Sec. V sets forth our conclusions.

\section{BOGOLIUBOV-DE GENNES EQUATIONS FOR AN NS JUNCTION}

The motion of elementary quasiparticles in an anisotropic superconductor can be described by the Bogoliubov-de Gennes equations ${ }^{11,12}$

$$
\begin{gathered}
\mathcal{H}_{e}(\mathbf{r}) u(\mathbf{r})+\int d \mathbf{r}^{\prime} \Delta\left(\mathbf{r}, \mathbf{r}^{\prime}\right) v\left(\mathbf{r}^{\prime}\right)=E u(\mathbf{r}), \\
-\mathcal{H}_{e}^{*}(\mathbf{r}) v(\mathbf{r})+\int d \mathbf{r}^{\prime} \Delta^{*}\left(\mathbf{r}, \mathbf{r}^{\prime}\right) u\left(\mathbf{r}^{\prime}\right)=E v(\mathbf{r}) .
\end{gathered}
$$

Here the excitation energy $E$ is measured relative to the Fermi energy $E_{F}$. The single-electron Hamiltonian is written as

$$
\mathcal{H}_{e}(\mathbf{r})=\frac{1}{2 m_{e}}\left(\frac{\hbar}{i} \nabla_{\mathbf{r}}+\frac{e \mathbf{A}(\mathbf{r})}{c}\right)^{2}+\hat{V}(\mathbf{r})-E_{F},
$$

where the vector potential $\mathbf{A}(\mathbf{r})$ is assumed to be zero in the following calculations, the potential energy $\hat{\mathbf{V}}(\mathbf{r})$ includes both the interaction between the electron and the external field and the interaction between the electron and the local nonmagnetic or magnetic scatterers. By introducing the center-of-mass coordinates $\mathbf{R}=\left(\mathbf{r}+\mathbf{r}^{\prime}\right) / 2$ and the relative coordinates $\mathbf{s}=\mathbf{r}-\mathbf{r}^{\prime}$, the Bogoliubov-de Gennes equations reduce to the Andreev equations in the WKBJ approximation ${ }^{2,12,13}$ if the potential varies slowly,

$$
\begin{aligned}
& E \widetilde{u}(\mathbf{r})=-i\left(\hbar^{2} / m_{e}\right) \mathbf{k}_{\mathbf{F}} \cdot \nabla \widetilde{u}(\mathbf{r})+\Delta\left(\hat{\mathbf{k}}_{F}, \mathbf{r}\right) \widetilde{v}(\mathbf{r}), \\
& E \widetilde{v}(\mathbf{r})=i\left(\hbar^{2} / m_{e}\right) \mathbf{k}_{\mathbf{F}} \cdot \nabla \widetilde{v}(\mathbf{r})+\Delta *\left(\hat{\mathbf{k}}_{F}, \mathbf{r}\right) \widetilde{u}(\mathbf{r}),
\end{aligned}
$$

where

$$
\left(\begin{array}{c}
\widetilde{u}(\mathbf{r}) \\
\widetilde{v}(\mathbf{r})
\end{array}\right)=e^{-i \mathbf{k}_{F} \cdot \mathbf{r}}\left(\begin{array}{l}
u(\mathbf{r}) \\
v(\mathbf{r})
\end{array}\right) .
$$

As shown in the Andreev equations, an unconventional superconducting pair potential depends not only on the centerof-mass coordinates $\mathbf{R}$ but also on the relative coordinates $\mathbf{S}$ in real space, or on the relative wave vector $\mathbf{k}$ after a Fourier transform, which in the weak-coupling theory is fixed on the Fermi surface such that only its direction $\hat{\mathbf{k}}_{F}=\mathbf{k}_{F} /\left|\mathbf{k}_{F}\right|$ is a variable. In particular, the pair potential of a bulk $d_{x_{a}^{2}-x_{b}^{2} \text {-wave superconductor can be written as }}^{5,14}$

$$
\Delta\left(\hat{\mathbf{k}}_{F}\right)=\Delta_{0}\left(\hat{k}_{x_{a}}^{2}-\hat{k}_{x_{b}}^{2}\right)=\Delta_{0}|\cos (2 \phi)| e^{i \varphi_{J}},
$$

where $\hat{k}_{x_{a}}=k_{x_{a}} /\left|\mathbf{k}_{F}\right|$ and $\hat{k}_{x_{b}}=k_{x_{b}} /\left|\mathbf{k}_{F}\right|$ are the normalized wave vector components along the $a$ and $b$ crystal axes of the $\mathrm{CuO}_{2}$ planes, $\phi$ is the azimuthal angle, and the gaugeinvariant phase is

$$
\varphi_{J}= \begin{cases}0, & \text { for } \cos (2 \phi)>0, \\ \pi, & \text { for } \cos (2 \phi)<0 .\end{cases}
$$

For a normal-metal-superconductor junction, due to the nonhomogeneity of the pair potential, quasiparticles (e.g., electronlike excitations) are partially reflected as electronlike and holelike excitations at the NS interface. Therefore, the effective pair potentials experienced by the electronlike excitations and holelike excitations are different from each other when the wave vectors associated with electron- and holelike excitations are different after the reflection. As shown in Eq. (2.5), if the order parameter in the superconductor has a $d$-wave symmetry, the effective pair potentials may have opposite signs under appropriate arrangements, which will never happen if the order parameter of the superconductor has an either isotropic or strongly anisotropic $s$-wave symmetry. We now assume the $x$ axis normal to the interface and the system is translationally invariant along the interface. The Andreev equations then assume the form

$$
E \widetilde{u}(x)=-i\left(\hbar^{2} / m_{e}\right) k_{F x}(d \widetilde{u}(x) / d x)+\Delta\left(\hat{\mathbf{k}}_{F x}, x\right) \widetilde{v}(x),
$$

$$
E \widetilde{v}(x)=i\left(\hbar^{2} / m_{e}\right) k_{F x}(d \widetilde{v}(x) / d x)+\Delta *\left(\hat{\mathbf{k}}_{F x}, x\right) \widetilde{u}(x) .
$$

By defining the interface at $x=0$ with the superconducting region at $x>0$, and neglecting the proximity effect, the pair potential for an NS junction can be given as

$$
\Delta\left(\hat{\mathbf{k}}_{F x}, x\right)=\Delta_{ \pm} \Theta(x) .
$$

Here $\Delta_{ \pm}$are the effective pair potentials experienced by the electronlike and holelike excitations, which take the form $\Delta_{ \pm}=\Delta_{0}$ for an isotropic $s$-wave superconductor, while take the form $\Delta_{ \pm}=\Delta_{0}|\cos (2 \theta \overline{+} 2 \alpha)| e^{i \varphi_{J_{ \pm}}}$for a $d$-wave supercon-

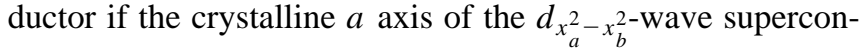
ductor, along which the magnitude of the pair potential reaches the maximum, is misoriented with an angle $\alpha$ with respect to the normal direction of the interface, and a beam of electrons are incident from the normal metal with an angle $\theta$ with respect to the normal direction of the interface. ${ }^{5,14}$ For our purpose to study the significant differences in conductance between $s$-wave and $d$-wave pairing symmetry cases, we particularly consider two special arrangements of the $d$-wave superconductor, i.e., $\alpha=\theta=0$ and $\alpha=\theta=\pi / 4$. For $\alpha=\theta=0, \quad \varphi_{J_{+}}=\varphi_{J_{-}}=0 ;$ for $\alpha=\theta=\pi / 4, \quad \varphi_{J_{+}}=0$ and $\varphi_{J_{-}}=\pi$. Notice that the results for the case $\alpha=\theta=0$ are similar to those for the pairing symmetry of the superconductor being of the isotropic $s$ wave. Therefore, in the following calculations, by fixing the gauge-invariant phase of the pair potential experienced by the electronlike excitations $\varphi_{J_{+}}=0$, we can use the gauge-invariant phase of the pair potential experienced by holelike excitations to specify the isotropic $s$-wave superconductor $\varphi_{J_{-}}=0$ and the special arrangement of the $d$-wave superconductor $\varphi_{J_{-}}=\pi$.

\section{I-V CHARACTERISTIC OF AN NS JUNCTION WITH A KONDO-LIKE MAGNETIC SCATTERING LAYER}

We model the Kondo-like magnetic interaction by a static scattering potential and the interaction potential between the electron spin and the spin of the scatterer, $\hat{V}(x)$ $=\left(V_{0}+V_{1} \mathbf{S}_{e} \cdot \mathbf{S}_{i}\right) \delta(x+L) .{ }^{15}$ Here $L$ is the distance of the 
scattering layer away from the NS interface, and the $\mathbf{S}_{e}$ and $\mathbf{S}_{i}$ denote, respectively, the spin operators of the electron and the scatterer. For simplicity, we assume the local spin $S_{i}=1 / 2$. Since the Bogoliubov-de Gennes equations are derived in the diagonal representation of electron spin operator $S_{e}^{2}$ and $S_{e z}$, the spin interaction operator $\mathbf{S}_{e} \cdot \mathbf{S}_{i}$ $=\left(\mathbf{S}^{2}-\mathbf{S}_{e}^{2}-\mathbf{S}_{i}^{2}\right) / 2$ with the total spin operator $\mathbf{S}=\mathbf{S}_{e}+\mathbf{S}_{i}$, should also be written in the product representation based on the eigenkets of $S_{e z}$ and $S_{i z}$. By making use of the relationship bewteen the triplet-singlet representation based on the eigenkets $\mathbf{S}^{2}$ and $S_{z}$ and the product representation,

$$
\begin{gathered}
|++\rangle \equiv\left|S=1, S_{z}=1\right\rangle, \\
|+-\rangle \equiv\left(\frac{1}{\sqrt{2}}\right)\left(\left|S=1, S_{z}=0\right\rangle+\left|S=0, S_{z}=0\right\rangle\right), \\
|-+\rangle \equiv\left(\frac{1}{\sqrt{2}}\right)\left(\left|S=1, S_{z}=0\right\rangle-\left|S=0, S_{z}=0\right\rangle\right),
\end{gathered}
$$

and

$$
|--\rangle \equiv\left|S=-1, S_{z}=-1\right\rangle,
$$

where $|++\rangle$ stands for $S_{e z}=1 / 2, S_{i z}=1 / 2$, and so forth, we are able to write $V_{0}+V_{1} \mathbf{S}_{e} \cdot \mathbf{S}_{i}$ as a $4 \times 4$ matrix,

$$
\begin{aligned}
V_{0}+V_{1} \mathbf{S}_{e} \cdot \mathbf{S}_{i} & =\left(\begin{array}{cccc}
V_{0}+V_{1} / 4 & 0 & 0 & 0 \\
0 & V_{0}-V_{1} / 4 & V_{1} / 2 & 0 \\
0 & V_{1} / 2 & V_{0}-V_{1} / 4 & 0 \\
0 & 0 & 0 & V_{0}+V_{1} / 4
\end{array}\right) .
\end{aligned}
$$

Correspondingly, the Bogoliubov-de Gennes equations are expanded into $8 \times 8$ matrix equations. Because the spin-flip process takes place only for those incident electrons forming the spin state $|+-\rangle$ or $|-+\rangle$ with the magnetic scatterer, we are able to decouple the problem into two kinds of scattering problem. The calculation of the contribution to the conductance from the non-spin-flip part is quite similar to that done by $\mathrm{Xu}$, Miller, and Ting. ${ }^{6}$ Here we concentrate on the calculation of the contribution from the spin-flip part. Define the wave function as four-component column vector $\Psi(x) \equiv\left(u_{+-}(x), u_{-+}(x), v_{+-}(x), v_{-+}(x)\right)^{\text {Trans }}$. When a beam of electrons incident from the normal conductor, which form the spin state $|+-\rangle$ with the spin scatterer, the general solution to the Andreev equations given by Eq. (2.7) is of the form

$$
\begin{aligned}
\Psi_{\mathrm{I}}= & \left(\begin{array}{l}
1 \\
0 \\
0 \\
0
\end{array}\right) e^{i q_{e}(x+L)}+\left(\begin{array}{c}
0 \\
0 \\
a_{+-} \\
a_{-+}
\end{array}\right) e^{i q_{h}(x+L)} \\
& +\left(\begin{array}{c}
b_{+-} \\
b_{-+} \\
0 \\
0
\end{array}\right) e^{-i q_{e}(x+L)}
\end{aligned}
$$

for $x<-L$;

$$
\begin{aligned}
\Psi_{\mathrm{II}} & =\left(\begin{array}{c}
f_{+-}^{(+)} \\
f_{-+}^{(+)} \\
0 \\
0
\end{array}\right) e^{i q_{e} x}+\left(\begin{array}{c}
f_{+-}^{(-)} \\
f_{-+}^{(-)} \\
0 \\
0
\end{array}\right) e^{-i q_{e} x}+\left(\begin{array}{c}
0 \\
0 \\
g_{+-}^{(+)} \\
g_{-+}^{(+)}
\end{array}\right) e^{i q_{h} x} \\
& +\left(\begin{array}{c}
0 \\
0 \\
g_{++-}^{(-)} \\
g_{-+}^{(-)}
\end{array}\right) e^{-i q_{h} x}
\end{aligned}
$$

for $-L<x<0$;

$$
\Psi_{\mathrm{III}}=\left(\begin{array}{c}
c_{+-} u_{0} \\
c_{-+} u_{0} \\
c_{-+} v_{0} \\
c_{+-} v_{0}
\end{array}\right) e^{i k_{e} x}+\left(\begin{array}{c}
d_{-+} v_{0} e^{i \varphi_{J_{-}}} \\
d_{+-} v_{0} e^{i \varphi_{J_{-}}} \\
d_{+-} u_{0} \\
d_{-+} u_{0}
\end{array}\right) e^{-i q_{e} x},
$$

for $0<x$. Here the "coherence factors" in the superconducting region are

$$
u_{0}^{2}=\frac{1}{2}\left(1+\frac{\sqrt{E^{2}-\Delta_{0}^{2}}}{E}\right), \quad v_{0}^{2}=\frac{1}{2}\left(1-\frac{\sqrt{E^{2}-\Delta_{0}^{2}}}{E}\right),
$$

respectively, and the wave vectors are determined from the dispersion relation,

$$
\begin{gathered}
q_{e, h}=k_{F x} \pm \frac{m_{e} E}{\hbar^{2} k_{F x}}, \\
k_{e, h}=k_{F x} \pm \frac{m_{e} \sqrt{E^{2}-\Delta_{0}^{2}}}{\hbar^{2} k_{F x}},
\end{gathered}
$$

where $k_{F x}=k_{F} \cos \theta$ with $\theta=0$ and $\pi / 4$. The Andreev reflection amplitudes $a_{+-}, a_{-+}$and the normal reflection amplitudes $b_{+-}, b_{-+}$can be obtained by imposing the following matching conditions on the wave function: 


$$
\Psi_{\mathrm{II}}(-L)=\Psi_{\mathrm{I}}(-L)
$$

$\Psi_{\mathrm{II}}^{\prime}(-L)-\Psi_{\mathrm{I}}^{\prime}(-L)$

$$
=\left(\frac{2 m_{e}}{\hbar^{2}}\right)\left[\left(V_{0}-V_{1} / 4\right) \hat{\mathbf{1}}+\left(V_{1} / 2\right) \hat{\boldsymbol{\tau}}\right] \Psi_{\mathrm{I}}(-L),
$$

and

$$
\begin{aligned}
& \Psi_{\mathrm{III}}(0)=\Psi_{\mathrm{II}}(0), \\
& \Psi_{\mathrm{III}}^{\prime}(0)=\Psi_{\mathrm{II}}^{\prime}(0) ;
\end{aligned}
$$

where $\hat{\mathbf{1}}$ is the $4 \times 4$ identity matrix and

$$
\hat{\boldsymbol{\tau}}=\left(\begin{array}{llll}
0 & 1 & 0 & 0 \\
1 & 0 & 0 & 0 \\
0 & 0 & 0 & 1 \\
0 & 0 & 1 & 0
\end{array}\right) .
$$

A little algebra yields the matrix equation determining the four reflection amplitudes

$$
\left(\begin{array}{cc}
\hat{\Pi}_{1} & \hat{\Pi}_{2} \\
\hat{\Pi}_{2} & \hat{\Pi}_{1}
\end{array}\right)\left(\begin{array}{c}
a_{-+} \\
b_{+-} \\
a_{+-} \\
b_{-+}
\end{array}\right)=\left(\begin{array}{c}
\left(1-i z_{2}\right) e^{i\left(q_{e}-q_{h}\right) L} \\
i z_{2} e^{-i\left(q_{e}-q_{h}\right) L} \\
-i z_{3} e^{i\left(q_{e}-q_{h}\right) L} \\
i z_{3} e^{-i\left(q_{e}-q_{h}\right) L}
\end{array}\right),
$$

where

$$
\begin{gathered}
\hat{\Pi}_{1}=\left(\begin{array}{cc}
\left(1-i z_{2}\right)\left(\frac{u_{0}}{v_{0}}\right) & i z_{2} e^{i\left(q_{e}-q_{h}\right) L} \\
i z_{2} e^{i \varphi_{J}}\left(\frac{v_{0}}{u_{0}}\right) & -\left(1+i z_{2}\right) e^{-i\left(q_{e}-q_{h}\right) L}
\end{array}\right), \\
\hat{\Pi}_{2}=\left(\begin{array}{cc}
-i z_{3}\left(\frac{u_{0}}{v_{0}}\right) & i z_{3} e^{i\left(q_{e}-q_{h}\right) L} \\
i z_{3} e^{i \varphi_{J}}\left(\frac{v_{0}}{u_{0}}\right) & -i z_{3} e^{-i\left(q_{e}-q_{h}\right) L}
\end{array}\right),
\end{gathered}
$$

with $z_{2}=\widetilde{V}_{0}-\widetilde{V}_{1} / 4, \quad z_{3}=\widetilde{V}_{1} / 2$, and $\widetilde{V}_{0}=m_{e} V_{0} / \hbar^{2} k_{F x}$, $\widetilde{V}_{1}=m_{e} V_{1} / \hbar^{2} k_{F x}$. To obtain Eq. (3.8), we have assumed that the Fermi energy is much greater than the pair potentials so that the difference between the wave vectors can be neglected except those appearing in exponents. It follows from Eq. (3.8) that the spin-flip process leads to the mixing effect in the tunneling of electrons between spin states $|+-\rangle$ and $|-+\rangle$, e.g., a beam of spin-up electrons will be partly Andreev reflected as spin-down and up holes, and partly reflected normally as spin-up and down electrons. We can also find the reflection amplitudes for non-spin-flip parts, i.e., $a_{++}, b_{++}$and $a_{--}, b_{--}$by simply replacing $z_{2}$ with $z_{1}=\widetilde{V}_{0}+\widetilde{V}_{1} / 4$ and setting $z_{3}=0$ in Eq. (3.8).

Within the framework of the BTK theory, ${ }^{10}$ the differential conductance is determined by the transmission coefficients. ${ }^{3,16}$ In the present case, we define the spin polarization of the electron and magnetic scatterer, $P_{e}=w_{e+}-w_{e-}$ and $P_{i}=w_{i+}-w_{i-}$, where $w_{e \pm}$ and $w_{i \pm}$

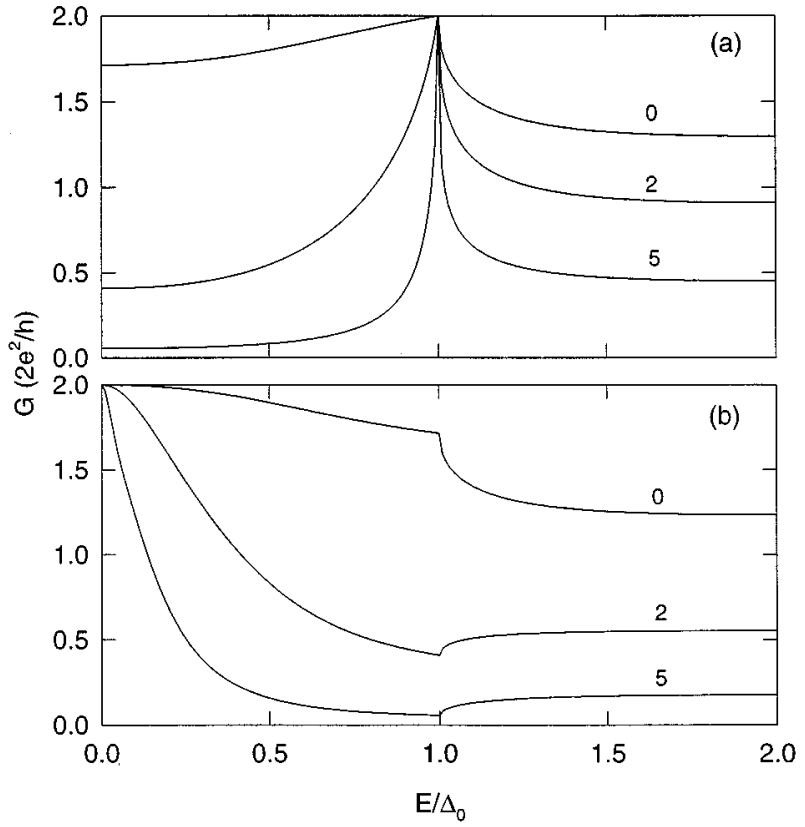

FIG. 1. Conductance versus $E / \Delta_{0}$ with various values of $\widetilde{V}_{1}=0,2,5$, for an $s$-wave NS junction (a) and a $d$-wave NS junction having a sign change of the order parameter (b). Here we take $L / \xi_{0}=0$ and assume the static scattering potential $\widetilde{V}_{0}=0.2$.

are, respectively, the probability for the electron and magnetic scatterer at spin up (down) state. By averaging over all possible spin states, we find the conductance at zero temperature to be

$$
\begin{aligned}
G= & \left(\frac{2 e^{2}}{h}\right)\left[\frac{1+P_{e} P_{i}}{2}\left(1+\left|a_{++}\right|^{2}-\left|b_{++}\right|^{2}\right)+\frac{1-P_{e} P_{i}}{2}\right. \\
& \left.\times\left(1+\left|a_{-+}\right|^{2}+\left|a_{+-}\right|^{2}-\left|b_{+-}\right|^{2}-\left|b_{-+}\right|^{2}\right)\right] \cdot
\end{aligned}
$$

Notice that $a_{++}(E)=a_{--}(E), b_{++}(E)=b_{--}(E)$, and the amplitudes $\left[a_{-+}(E), a_{+-}(E)\right],\left[b_{+-}(E), b_{-+}(E)\right]$ for incident electrons forming spin state $|+-\rangle$ with the spin scatterer correspond one by one to those $\left[a_{+-}(E), a_{-+}(E)\right]$, $\left[b_{-+}(E), b_{+-}(E)\right]$ for those incident electrons forming spin state $|-+\rangle$ with the spin scatterer. If both the incident electron and the spin scatterer are spin up or both spin down, i.e., $P_{e}=P_{i}=1$ or $P_{e}=P_{i}=-1$, the contribution to the conductance is all from the non-spin-flip process. If the electron and the spin scatterer are spin polarized with opposite directions, i.e., $P_{e}=-P_{i}=1$ or $P_{e}=-P_{i}=-1$, then all contributions to the conductance comes from the spin-flip process. It is the contribution from the spin-flip part that may lead to the conductance behavior different from that of an NS junction with nonmagnetic scattering. Here we are interested in the spinunpolarization case, in which $P_{e}=P_{i}=0$ and the conductance is thus one half of the sum of conductance in above two limiting cases.

In Figs. 1 and 2, we plot the differential conductance of an isotropic $s$-wave NS junction and a $d$-wave NS junction under the arrangement such that $\alpha=\theta=\pi / 4$. The distances between the Kondo-like magnetic scattering layer and the NS interface are $L=0$ and $L=5 \xi_{0}$ with the superconducting 


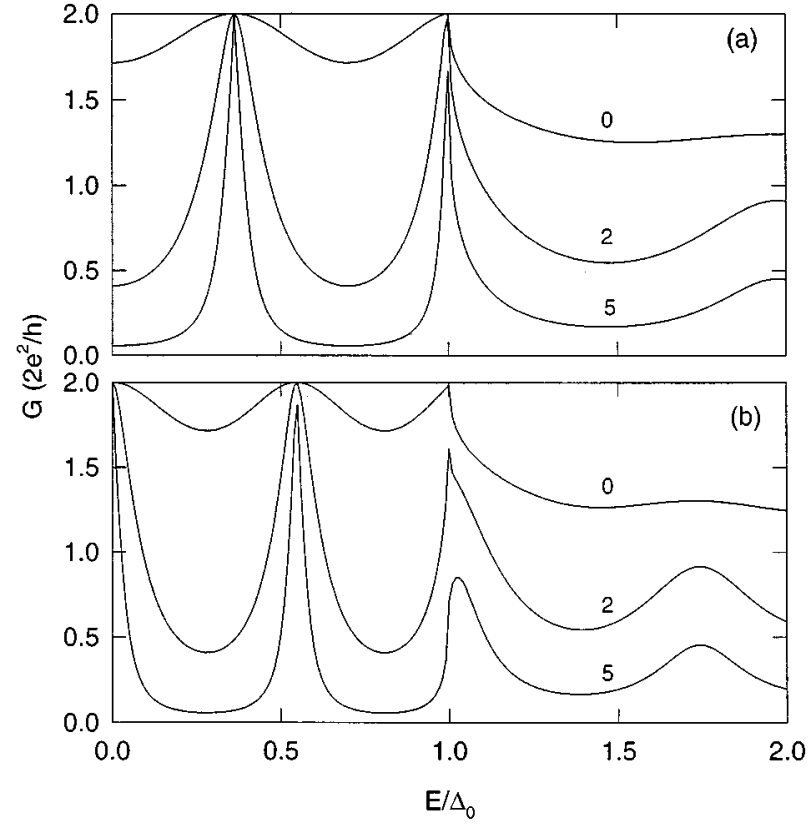

FIG. 2. Same as Fig. 1 but with $L / \xi_{0}=5$.

coherence length being defined as $\xi_{0}=\hbar v_{F} / \pi \Delta_{0}$. In addition, the strength of the static scattering potential $\widetilde{V}_{0}=0.2$ is fixed. As shown in Fig. 1, we can see that when the scattering layer is located at the NS interface, as the spin-flip scattering increases, the conductance exhibits a sharp peak at the bias voltage $\Delta_{0} / e$ for an isotropic $s$-wave NS junction; however, for a $d$-wave NS junction, the conductance peak takes place at zero bias due to the sign change of the order parameter. If the scattering layer is away from the NS interface by serveral superconducting coherence lengths, new subgap resonances develop but the bias of conductance anomaly does not change. All of these results show clearly that the ZBA which was attributed to the $d$-wave order parameter can still survive in the presence of the local Kondo-like magnetic scattering. It seems in this sense that by identifying the relative positions for a normal-metal-unconventional superconductor junction with respect to those for a normal-metalconventional superconductor junction, as suggested by previous work, ${ }^{5,6}$ can provide distinct signatures of pairing symmetry in high- $T_{c}$ superconductors if we do not consider the ferromagnetic scattering effect, which will be studied below.

\section{I-V CHARACTERISTIC OF AN NS JUNCTION WITH A FERROMAGNETIC SCATTERING LAYER}

Another kind of magnetic scattering effect we consider is the short-range exchange interaction between electrons and a local ferromagnetic layer, which could be written as

$$
\hat{V}=V_{\mathrm{Ferr}} \sigma_{z} \delta(x+L),
$$

where $\sigma_{z}$ is the $z$ component of the Pauli matrix,

$$
\sigma_{z}=\left(\begin{array}{cc}
1 & 0 \\
0 & -1
\end{array}\right)
$$

This model may be applicable when the exchange interaction is the dominant magnetic effect on the transport properties through a normal-metal-superconductor junction. In this situation, the matching condition for the two-component wave functions $\Psi(x)=(u(x), v(x))^{\text {Trans }}$ at the scattering layer reads

$$
\begin{gathered}
\Psi\left(-L+0^{+}\right)=\Psi\left(-L-0^{+}\right), \\
\left.\frac{d \Psi(x)}{d x}\right|_{x=-L+0^{+}}-\left.\frac{d \Psi(x)}{d x}\right|_{x=-L-0^{+}}= \pm \frac{2 m_{e} V_{\mathrm{Ferr}}}{\hbar^{2}} \Psi(-L),
\end{gathered}
$$

where the signs " \pm " depend on the spin state of incident electrons, " +" for spin up and " - " for spin down. It is not difficult to understand this matching condition by noting that the incoming electron and the Andreev-reflected hole pertain to opposite spin states. For recent several years, there has been both experimental and theoretical work on tunneling through superconductor-ferromagnetic insulatorsuperconductor (SFiS) junctions. ${ }^{17}$ More recently, there has also been a theoretical work on the transport properties in a ferromagnet-superconductor junction with (FFS) or without a tunnel barrier (FS) ${ }^{18}$ where polarization of the current comes from the different density of states of spin-up and spin-down conduction electrons in the ferromagnet. However, in the normal-metal-ferromagnetic insulator-normalmetal-superconductor (NFiNS) junction, the spinpolarization effect stems from the different barriers heights experienced by electrons at different spin state in the magnetic scattering layer. By applying the BTK theory to our NFiNS case, the conductance is now given by ${ }^{18}$

$$
G=\frac{e^{2}}{h} \sum_{\sigma=+,-}\left(1+\left|r_{h \bar{\sigma}, e \sigma}\right|^{2}-\left|r_{e \sigma, e \sigma}\right|^{2}\right),
$$

which shows clearly that an incoming electron of spin $\sigma$ is normally reflected as an electron of the same spin $\sigma$ and Andreev reflected as a hole of the opposite spin $\bar{\sigma}$. As a result, we give the amplitudes of normal reflection $r_{e+, e+}$ and Andreev reflection $r_{h-, e+}$

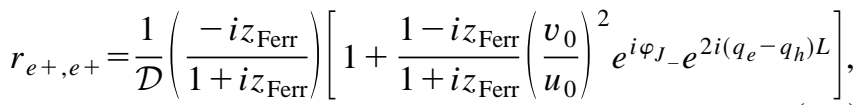

and

$$
r_{h-, e+}=\frac{1}{\mathcal{D}}\left(\frac{v_{0}}{u_{0}}\right) \frac{1}{\left(1+i z_{\mathrm{Ferr}}\right)^{2}} e^{i\left(q_{e}-q_{h}\right) L},
$$

where $z_{\mathrm{Ferr}}=m_{e} V_{\mathrm{Ferr}} / \hbar^{2} k_{F x}$ and

$$
\mathcal{D}=1+\frac{z_{\text {Ferr }}^{2}}{\left(1+i z_{\text {Ferr }}\right)^{2}}\left(\frac{v_{0}}{u_{0}}\right)^{2} e^{i \varphi_{J}} e^{2 i\left(q_{e}-q_{h}\right) L} .
$$

As for $r_{e^{-, e-}}$ and $r_{h+, e-}$, they can be obtained by replacing $z_{\text {Ferr }}$ with $-z_{\text {Ferr }}$. We plot the conductance as a function of energy with a variety of values of $z_{\text {Ferr }}$ in Figs. 3-5 with $L / \xi_{0}=0,2,5$, respectively. As shown in Fig. 3, when the ferromagnetic scattering layer is located at the NS interface, at small values of the scattering length, the conductance behavior in normal-metal-ferromagnet- $s$-wave superconductor 


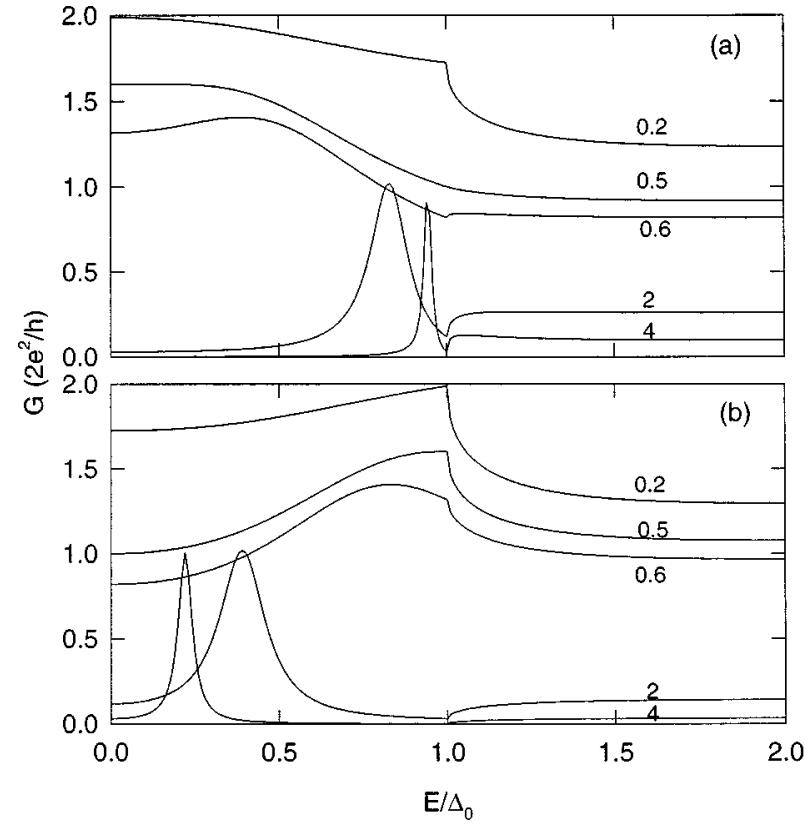

FIG. 3. Conductance versus $E / \Delta_{0}$ with various values of $z_{\text {Ferr }}=0.2,0.5,0.6,2,4$, for an $s$-wave NS junction (a) and a $d$-wave NS junction having sign change of the order parameter (b). Here $L / \xi_{0}=0$.

junctions is quite similar to that in normal-metal- $d$-wave superconductor junctions with nonmagnetic tunnel barrier; while the conductance behavior in normal-metalferromagnetic- $d$-wave superconductor junctions is quite similar to that in normal-metal- $s$-wave superconductor junctions containing a nonmagnetic tunnel barrier. This result is particularly important and we believe that the presence of a low-level ferromagnetic scattering could make a direct experimental determination of the pairing symmetry via the

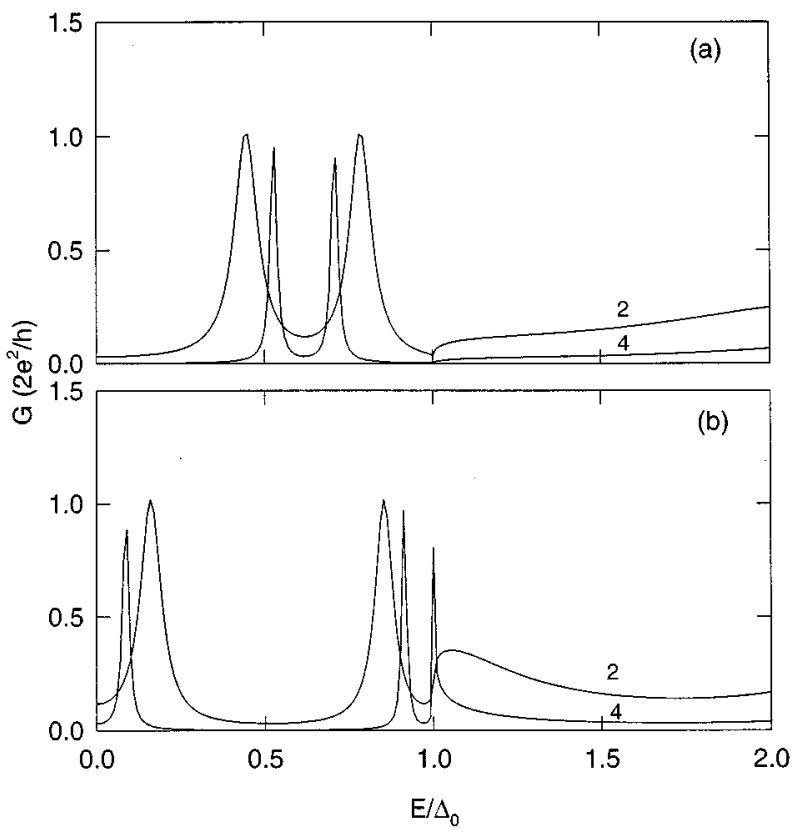

FIG. 4. Same as Fig. 3 but with $L / \xi_{0}=2$ and $z_{\text {Ferr }}=2,4$.

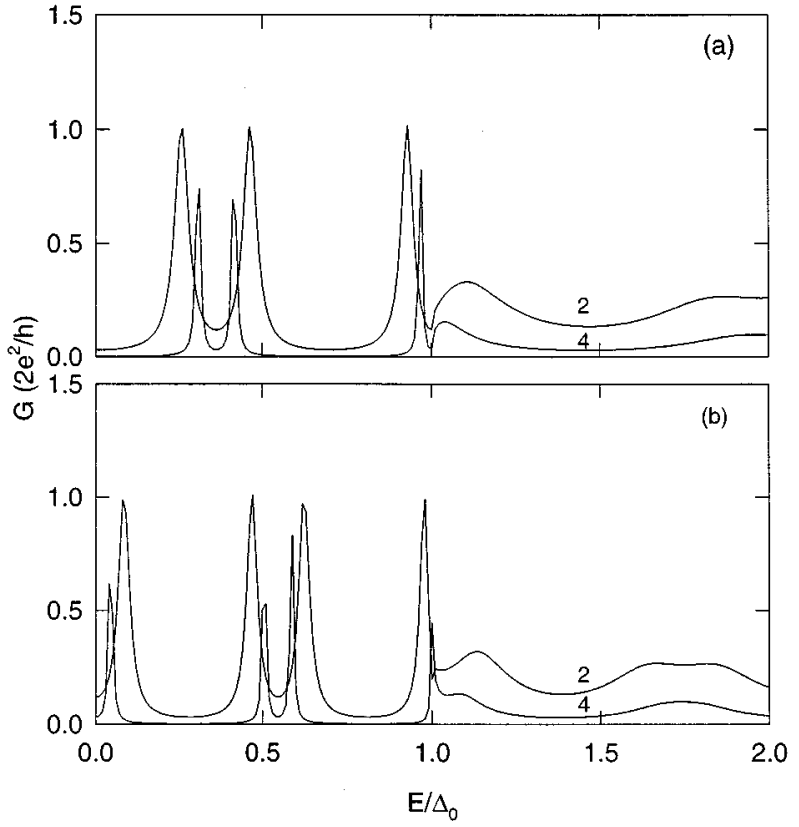

FIG. 5. Same as Fig. 3 but with $L / \xi_{0}=5$ and $z_{\text {Ferr }}=2,4$.

electron tunneling through an NS junction extremely hazardous. Also intriguingly, as the strength of the ferromagnetic scattering potential increases, a conductance peak is induced at the finite bias for both $s$-wave and $d$-wave cases. However, the conductance peak for the $s$-wave case is shifted toward the energy-gap position, while that for $d$-wave case is shifted to the zero bias. When the ferromagnetic scattering layer is placed in the normal metal by several superconducting coherence lengths away from the interface, due to the interference effect arising from the normal reflection at the ferromagnetic layer and the Andreev reflection at the interface, new subgap resonances appear in the conductance (see Figs. 4 and 5). Moreover, because of the different effective potentials experienced by the electrons of different spin states, the main subgap resonance peaks appearing in the conductance for a normal-metal-superconductor junction with nonmagnetic scattering potential are now splitted twofoldly. In particular, as the strength of the exchange interaction increases, breaking peaks of each pair approach nearby for the $s$-wave case while become far away for the $d$-wave case. In fact, these subgap resonances come from the formation of quasibound states inside the energy gap between the barrier and the interface, which are determined by the poles of the current transmission. ${ }^{6}$ Setting $\mathcal{D}=0$, we obtain

$$
\frac{z_{\mathrm{Ferr}}^{2}}{\left(1 \pm i z_{\mathrm{Ferr}}\right)^{2}}\left(\frac{v_{0}}{u_{0}}\right)^{2} e^{i \varphi_{J_{-}}} e^{2 i\left(q_{e}-q_{h}\right) L}=-1
$$

where the " + ", sign is for electrons of spin up and the " - " sign is for those of spin down. As in the treatment in Ref. 6, we take $E=E_{R}+i E_{I}$ for the quasibound states determined by Eq. (4.6), where $E_{R}$ is the quasibound state energy and $E_{I}$ is the escape rate of quasiparticles from this quasibound state. The approximate formulas for the two quantities are found to be 


$$
\begin{gathered}
\frac{2}{\pi}\left(\frac{E_{R}}{\Delta_{0}}\right)\left(\frac{L}{\xi_{0}}\right)-\cos ^{-1}\left(\frac{E_{R}}{\Delta_{0}}\right) \mp \varphi_{0} \approx\left(n+\frac{1}{2}\right) \pi, \\
E_{I} \approx \Delta_{0}\left(\frac{\pi \xi_{0}}{4 L}\right) \ln \frac{z_{\mathrm{Ferr}}^{2}}{1+z_{\mathrm{Ferr}}^{2}},
\end{gathered}
$$

for an $s$-wave NS junction, and

$$
\begin{gathered}
\frac{2 \sqrt{2}}{\pi}\left(\frac{E_{R}}{\Delta_{0}}\right)\left(\frac{L}{\xi_{0}}\right)-\cos ^{-1}\left(\frac{E_{R}}{\Delta_{0}}\right) \mp \varphi_{0} \approx n \pi, \\
E_{I} \approx \Delta_{0}\left(\frac{\pi \xi_{0}}{4 \sqrt{2} L}\right) \ln \frac{z_{\mathrm{Ferr}}^{2}}{1+z_{\mathrm{Ferr}}^{2}},
\end{gathered}
$$

for a $d$-wave NS junction, where $\varphi_{0}=\cos ^{-1}\left(1 / \sqrt{1+z_{\text {Ferr }}^{2}}\right)$ is the phase shift which is crucial to distinguish the ferromagnetic scattering effect from both the nonmagnetic scattering and the Kondo-like magnetic scattering effect on the conductance behavior of a normal-metal-superconductor junction. We can also see clearly that this phase shift leads to the resonance peak splitting in the conductance. To study this effect in some detail, we discuss the case, as an illustration, that the ferromagnetic scattering layer is located at the interface $(L \rightarrow 0)$. In this limiting case, we have $E_{R}= \pm \Delta_{0} \sin \varphi_{0}$ for an $s$-wave NS junction and $E_{R}= \pm \Delta_{0} \cos \varphi_{0}$ for a $d$-wave NS junction. Therefore, if the strength of the ferromagnetic barrier is finite and $\varphi_{0}$ is thus finite, neither midgap states at the Fermi surface $\left(E_{R}=0\right)$ for the $d$-wave NS junction nor gap states $\left(E_{R}=\Delta_{0}\right)$ at the energy gap for the $s$-wave junction exist. Only when the ferromagnetic layer is completely insulating $\left(z_{\mathrm{Ferr}} \rightarrow \infty\right.$ and $\left.\varphi_{0}=\pi / 2\right)$, can the midgap states and gap states be exhibited, respectively, in the above-mentioned junctions.

\section{CONCLUSIONS}

We have calculated the conductance through a normalmetal- $d$-wave superconductor junction as well as a normalmetal-s-wave superconductor junction, which contains a sheet Kondo-like magnetic scattering layer or a ferromagnetic scattering layer. It is shown that the conductance behavior in the presence of a Kondo-like magnetic scattering layer is quite similar to that in the presence of a nonmagnetic barrier, although the spin-flip process takes place in the former case. More importantly, we have shown that in the presence of ferromagnetic scattering, if the layer is located at the NS interface, the conductance of a $d$-wave NS junction containing a ferromagnetic barrier resembles that of an $s$-wave junction with a nonmagnetic barrier, and the conductance of an $s$-wave NS junction with a ferromagnetic barrier resembles that of a $d$-wave junction involving a nonmagnetic layer. This result may complicate significantly the conclusive determination of the pairing symmetry in high- $T_{c}$ superconductors by the quasiparticle tunneling into the superconductor. Relative to the nonmagnetic scattering case, the resonance peak splitting in the conductance is exhibited when the layer is several superconducting coherence lengths away from the NS interface.

Finally, we would like to make a remark: As we have done in this work, to apply the one-dimensional description to the three-dimensional case requires that the system should have the translational invariance along the interface perpendicular to the transport direction. Realistically, the Kondo exchange coupling arises from the magnetic atoms having spin, which breaks the translational invariance. Therefore, the interaction term we have taken in Sec. III is a simple theoretical model and the discussion there is more relevant to a real one-dimensional system. Nevertheless, we notice that, the Kondo-like magnetic scattering effect described by our model is stronger than that in the practical three-dimensional case. Therefore, we can expect that no ZBA in conductance is induced by the Kondo impurities in practical threedimensional systems. On the other hand, when the density of magnetic atoms in the ferromagnet layer is very high, these atoms are so strongly coupled to one another that the magnetic spins align parallel to the interface. In this case, the magnetization field will be carried entirely within the thin layer. Consequently, our one-dimensional description of the ferromagnetic scattering effect could be applied to the threedimensional case, which is also more easily realized by experiments.

\section{ACKNOWLEDGMENTS}

We would like to thank Professor C. S. Ting for initiating this work. We are also grateful to Professor C. W. J. Beenakker for helpful discussions. This work was supported by the RGC grant of Hong Kong under Grant No. HKU262/95P and the CRCG grant at the University of Hong Kong.
${ }^{1}$ For a review, see, C. W. J. Beenakker, in Mesoscopic Quantum Physics, edited by E. Akkermans, G. Montambaux, and J.-L. Pichard (North-Holland, Amsterdam, 1995); T. M. Klapwijk, Physica (Amsterdam) 197B, 481 (1994).

${ }^{2}$ A. F. Andreev, Zh. Eksp. Teor. Fiz. 46, 1823 (1964) [Sov. Phys. JETP 19, 1228 (1964)]; 49, 655 (1965) [22, 455 (1966)]; 51, 1510 (1966) [24, 1019 (1967)].

${ }^{3}$ C. W. J. Beenakker, Phys. Rev. B 46, 12841 (1992).

${ }^{4}$ For a review, see, D. J. Scalapino, Phys. Rep. 250, 329 (1995); M. Sigrist and T. M. Rice, Rev. Mod. Phys. 67, 503 (1995); D. J. van Harlingen, ibid. 67, 515 (1995); B. G. Levi, Phys. Today 49 (1), 19 (1996).
${ }^{5}$ S. Kashiwaya, Y. Tanaka, M. Koyanagi, H. Takashima, and K. Kajimura, Phys. Rev. B 51, 1350 (1995); Y. Tanaka and S. Kashiwaya, Phys. Rev. Lett. 74, 3451 (1995).

${ }^{6}$ J. H. Xu, J. H. Miller, and C. S. Ting, Phys. Rev. B 53, 3604 (1996).

${ }^{7}$ Jian-Xin Zhu, Z. D. Wang, D. Y. Xing, and Z. C. Dong, Physica (Amsterdam) 260C, 217 (1996).

${ }^{8}$ For a review of the ZBA in high- $T_{c}$ superconductor junctions, see T. Walsh, Int. J. Mod. Phys. B 6, 125 (1992).

${ }^{9}$ C. R. Hu, Phys. Rev. Lett. 72, 1526 (1994).

${ }^{10}$ G. E. Blonder, M. Tinkham, and T. M. Klapwijk, Phys. Rev. B 25, 4515 (1982). 
${ }^{11}$ P. G. de Gennes, Superconductivity of Metals and Alloys (Benjamin, New York, 1966).

${ }^{12}$ Chr. Bruder, Phys. Rev. B 41, 4017 (1990).

${ }^{13}$ J. Kurkijärvi and D. Rainer, in Modern Problems in Condensed Matter Sciences, edited by W. P. Halperin and L. P. Pitaerskii (Elsevier, Amsterdam, 1989).

${ }^{14}$ Jian-Xin Zhu, Z. D. Wang, and H. X. Tang, Phys. Rev. B 54, 7354 (1996); H. X. Tang, Z. D. Wang, and Jian-Xin Zhu, ibid. 54, 12509 (1996).

${ }^{15}$ In this model, the electron-electron correlation mediated by the local spin has been neglected. This requires that the temperature remain above the Kondo temperature. However, the temperature must be low enough to maintain the electronic phase coherence in the system. Such a temperature range is experimentally accessible.

${ }^{16}$ Y. Takane and H. Ebisawa, J. Phys. Soc. Jpn. 60, 3130 (1991); 61, 1685 (1992).

${ }^{17}$ F. Stageberg, R. Cantor, A. M. Goldman, and G. B. Arnold, Phys. Rev. B 32, 3292 (1985); M. J. Deweert and G. B. Arnold, Phys. Rev. Lett. 55, 1522 (1985); Phys. Rev. B 39, 11307 (1989).

${ }^{18}$ M. J. M. de Jong and C. W. J. Beenakker, Phys. Rev. Lett. 74, 1657 (1995). 